\title{
Women, endometriosis, and the evolution of imaging
}

\section{Suzan Menasce Goldman ${ }^{1}$}

My first contact with endometriosis was when I was about 10 years old and I heard my mother commenting to her friends "Do you know that pain that Clarinha has had for years? They finally found out that she has something called endometriosis! The doctor said that it is very rare and means that a piece of the uterus has grown out of place ..." I was a bit scared of that "weird disease", and its name was stored in my memory.

Until the 1990s, when laparoscopy came to be widely used, endometriosis was still relatively unknown. Laparoscopy revolutionized the diagnosis of endometriosis, and, from that time onward, understanding of the disease has increased greatly. Concomitantly, we radiologists began to walk side-byside with gynecologists, initially in relation to the diagnosis of ovarian endometriomas, whose presentations on ultrasound became "typical", with the famous "chocolate cysts". Now we have become a player. How many women have benefited from these findings? Thousands! One of the first studies, published in 1989, showed that magnetic resonance imaging (MRI) has good sensitivity for the characterization of endometriomas, although it did not replace laparoscopy ${ }^{(1)}$.

In 1993, the classic study conducted by Sugimura et al. ${ }^{(2)}$ defined the signal behavior of endometriomas on MRI, the famous T2 shading, which defined the pattern of different shades of gray on T2-weighted images and could indicate different epochs of bleeding. In 1994, despite so many advances, endometriosis was still considered an enigma in terms of its diagnosis and its origin ${ }^{(3)}$. The evolution of imaging allowed radiologists to be protagonists in so many other diseases, so why were we still playing only a supporting role in endometriosis? It was because there was still a lack of understanding of the imaging aspects of deep endometriosis in the main imaging methods applied for pelvic assessment.

Ultrasound soon proved to be the best imaging method for the evaluation of endometriosis, and, within a few years, the best examination technique and key imaging features for the assessment of deep foci had been defined. In 1985, the

1. Associate Professor, Department of Imaging Diagnosis, Escola Paulista de Medicina da Universidade Federal de São Paulo (EPM-Unifesp), São Paulo, SP, Brazil. E-mail: smgold@terra.com.br.

https://orcid.org/0000-0002-3596-3419. efficacy of ultrasound was only $10 \%$ of that of laparoscopy ${ }^{(\mathbf{4})}$. After the examination technique (bowel preparation, specific maneuvers, and systematic, compartmentalized evaluation) was defined, the efficacy of ultrasound rose to approximately 95\% ${ }^{(5,6)}$. In 2009, Chapron et al. recommended that transvaginal ultrasound (TVUS) with bowel preparation be used as the first-line examination in the preoperative evaluation of patients with endometriosis ${ }^{(7)}$. In this issue of Radiologia Brasileira, there is an excellent, comprehensive article describing the presentation forms of endometriosis on TVUS with bowel preparation ${ }^{(\mathbf{8})}$.

Although MRI evolved in parallel with ultrasound, it did so more slowly. In the early 2000s, when ultrasound was already emerging as a promising method for the evaluation of endometriosis, MRI struggled with protocol variability, equipment quality, and, consequently, image quality. As image quality improved and protocols evolved, the efficacy of MRI improved greatly, coming to equal that of TVUS, as demonstrated in a meta-analysis published in $2018^{(9)}$.

With all of this favorable evolution, imaging came to play a leading role in the evaluation of endometriosis. It has now replaced laparoscopy in the diagnosis and in the planning of treatment (clinical or surgical), as well as allowing the safe monitoring of the affected patients.

\section{REFERENCES}

1. Zawin M, McCarthy S, Scoutt L, et al. Endometriosis: appearance and detection at MR imaging. Radiology. 1989;171:693-6.

2. Sugimura K, Okizuka H, Imaoka I, et al. Pelvic endometriosis: detection and diagnosis with chemical shift MR imaging. Radiology. 1993;188:435-8.

3. Thomas EJ. Endometriosis: still an enigma. Br J Obstet Gynaecol. 1993;100:6157.

4. Friedman $\mathrm{H}$, Vogelzang RL, Mendelson EB, et al. Endometriosis detection by US with laparoscopic correlation. Radiology. 1985;157:217-20.

5. Guerriero S, Saba L, Pascual MA, et al. Transvaginal ultrasound vs magnetic resonance imaging for diagnosing deep infiltrating endometriosis: systematic review and meta-analysis. Ultrasound Obstet Gynecol. 2018;51:586-95.

6. Fonseca EKUN, Bastos BB, Yamauchi FI, et al. Ruptured endometrioma: main imaging findings. Radiol Bras. 2018;51:411-2.

7. Piketty M, Chopin N, Dousset B, et al. Preoperative work-up for patients with deeply infiltrating endometriosis: transvaginal ultrasonography must definitely be the firstline imaging examination. Hum Reprod. 2009;24:602-7.

8. Oliveira JGA, Bonfada V, Zanella JFP, Coser J. Ultrassonografia transvaginal na endometriose profunda: ensaio iconográfico. Radiol Bras. 2019;52:337-41.

9. Oliveira JGA, Bonfada V, Figueiró MF, et al. Clinical features, socio-demographic profile and ultrasound findings in women with endometriosis symptoms. Sci Med. 2018;28(4):ID30496.

\section{$(c)) \mathrm{EY}$}

